\title{
RELACIÓN ENTRE RENTABILIDAD Y ADHESIÓN AL PACTO GLOBAL: UN ANÁLISIS DE EMPRESAS COLOMBIANAS
}

\author{
RELATIONSHIP BETWEEN PROFITABILITY AND \\ ADHERENCE TO THE GLOBAL COMPACT: \\ AN ANALYSIS OF COLOMBIAN COMPANIES
}

\section{RESUMEN}

El propósito de este documento es establecer el efecto que tiene sobre la rentabilidad la pertenencia al Pacto

\section{Liliana Elizabeth Ruiz Acosta' David Andrés Camargo Mayorga² Octavio Cardona García ${ }^{3}$}

1 Contador público y magíster en Gestión Empresarial. Docente investigador de tiempo completo y miembro del grupo GECS de la Facultad de Ciencias Económicas de la Universidad Militar Nueva Granada.

Correo electrónico: liliana.ruiz@unimilitar.edu.co

Orcid: https://orcid.org/0000-0003-3323-8480

2 Economista, magíster en Economía y en Educación. Docente Investigador de tiempo completo y miembro del grupo de Estudios Contemporáneos en Contabilidad, Gestión y Organizaciones de la Facultad de Ciencias Económicas de la Universidad Militar Nueva Granada.

Correo electrónico: david.camargo@unimilitar.edu.co

Orcid: https://orcid.org/0000-0002-5290-8251

3 Asistente de investigación de la Universidad Militar Nueva Granada. Bogotá, Colombia.

Correo electrónico: octavio.cardona.g@gmail.com

Orcid: https://orcid.org/0000-0001-6257-5270

Código JEL: C58, M14, M41.

Fecha de recepción: 23/08/2018

Fecha de aceptación: 26/09/2018

DOI: https://doi.org/10.18601/16577175.n22.10 
2017, encontrándose que 110 empresas son adheridas al PG. La metodología es cuantitativa y se utilizaron dos métodos estadísticos para establecer las diferencias entre la rentabilidad del grupo de empresas que pertenecen al PG y las que no. Los hallazgos no son concluyentes para mostrar una relación estadísticamente significativa entre las variables analizadas.

Palabras clave: activos, beneficio neto, estadística, gestión financiera, sostenibilidad.

\section{ABSTRACT}

The purpose of this document is to establish the effect on the profitability of belonging to the Global Compact (GC) in the member companies. For this, the profitability was calculated for 1,000 Colombian companies that reported accounting information to the "Superintendencia de Sociedades" in 2017, finding from this that 110 are adhered to the GC. The methodology is quantitative and two statistical methods were used to establish the differences between the profitability of the group of companies that belong to the GC, and those that do not. The findings are not conclusive to show a statistically significant relationship between the variables analyzed.

Keywords: assets, net profit, statistics, financial management, sustainability.

\section{INTRODUCCIÓN}

Las empresas se suelen ver atraídas por las ganancias potenciales que pueden percibir al entrar a un mercado o al comportarse de alguna manera en particular. Según Demsetz (1997), el accionar empresarial se basa simplemente en los incentivos que ofrecen los precios relativos, los que para Coase (1994) se miden en dinero (o aun sin este), motivando a los empresarios a elegir.

Hacer Responsabilidad Social $\left(\mathrm{RS}^{4}\right)$ es una elección, no una imposición. Emprender acciones socialmente responsables significa ser sensible a lo que pasa con la sociedad y el ambiente, lo que en palabras de Olcese, Rodríguez y Alfaro (2008) es la capacidad de una firma para crear valor para sus grupos de incidencia.

Adherirse al Pacto Global (PG) es una acción empresarial que demuestra compromiso con prácticas social y ambientalmente responsables. El PG es una iniciativa de la Organización de Naciones Unidas (ONU), que tiene sus orígenes en las etapas de "reconocimiento" y "compromiso" de la RS a nivel global en la década de los 70 (Yepes, Peña y Sánchez, 2007). La adhesión de una empresa supone el cumplimento tanto de los diez Objetivos de Desarrollo del Milenio como de los diecisiete Objetivos de Desarrollo Sostenible. 
Descrito lo anterior, surge una pregunta: ¿mejorarán su rentabilidad las empresas que se adhieren al PG? En respuesta a este interrogante, el presente artículo analiza cómo la rentabilidad de los activos (ROA) de 1000 empresas, que reportaron en el 2017 información de sus balances y estados financieros a la Superintendencia de Sociedades, cambia si se suscribieron o no al PG.

Este documento inicia con esta introducción, sigue con una revisión de literatura sobre el tema, la descripción de los materiales y métodos utilizados, la presentación de los resultados, y, finaliza, con las conclusiones.

\section{REVISIÓN DE LITERATURA}

Las empresas hacen RS motivadas por muchas razones, incluyendo la mejora de su desempeño financiero. Para Drucker (1984), cuando una compañía transforma un problema social en una rentable oportunidad de negocio, ofreciendo puestos de trabajo bien remunerados y permite la acumulación de riqueza, está haciendo RS. Para Friedman (1970), la responsabilidad de las empresas es casi que exclusiva con las ganancias para sus socios, aflorando los valores económicos como criterio de cuantificación del éxito (Bour, 2012). Del mismo modo, fabricar, distribuir y comercializar productos que se venden a consumidores en el mercado (Liya y Shaohong, 2013) es también RS. Según esto, las empresas por el simple hecho de existir son responsables con el entorno social que las circunda, independientemente de si son o no rentables.
Además, se imputan a la RS otras motivaciones para las compañías, las cuales tienen que ver con la gestión de la imagen corporativa (Valenzuela, Jara-Bertin y Villegas, 2015), la ética ambiental, entre otras cuestiones.

Esto condiciona a la empresa, porque según Duque, Cardona y Rendón (2014), esta siente como las reglas de contratación, relacionamiento comercial e intercambio en el mercado convierten en obligatorias las prácticas de RS, que a priori son de carácter voluntario, pero que en últimas aseguran que la empresa sobreviva. Esto sin duda modifica el entorno competitivo (Nieto y Fernández, 2004).

Lo anterior desdibuja la esencia del RS, le quita su voluntariedad, porque como lo expresa la Comisión Europea (2001), las decisiones empresariales deberían propender por mejorar el ambiente y la sociedad. La tabla 1 muestra un resumen de la revisión de literatura, en la que se compilan estudios que han relacionado la RS con el desempeño financiero.

Para instrumentalizar la RS se han proliferado estándares que se representan en normas, lineamientos, guías, modelos, entre otros (Duque, Cardona y Rendón, 2014). Uno de estos intentos por instrumentalizar la RS es el Pacto Global de la ONU, el cual se explica según sus dimensiones y principios en la tabla 2.

Los principios del PG, listados en la tabla 2, los cumplen las compañías adheridas, cuando implementan un sistema de gestión que comprende una fase de 
Tabla 1.

Revisión de literatura

\begin{tabular}{|c|c|c|}
\hline Autores & Descripción & $\begin{array}{l}\text { Relación entre } \\
\text { RS y desempe- } \\
\text { ño financiero }\end{array}$ \\
\hline Preston y O’Bannon (1997) & $\begin{array}{l}\text { Estudio correlacional de } 67 \text { grandes cor- } \\
\text { poraciones de Estados Unidos en el pe- } \\
\text { riodo 1982-1992. Los hallazgos respaldan } \\
\text { la hipótesis de que los grupos de interés } \\
\text { inciden en los resultados financieros de } \\
\text { las empresas. Se concluye que el desem- } \\
\text { peño financiero es más fuerte cuando está } \\
\text { antecedido de desempeño social. }\end{array}$ & Positiva. \\
\hline Walman et al. (2006) & $\begin{array}{l}\text { Trabajo longitudinal que analizó } 561 \\
\text { empresas de quince países en los cinco } \\
\text { continentes. Su principal hallazgo es } \\
\text { que la relación entre RSE y desempeño } \\
\text { financiero cambia por la variable cultura. }\end{array}$ & Positiva. \\
\hline Makni, Francoeur y Bellavance (2009) & $\begin{array}{l}\text { Estudio para } 179 \text { empresas canadienses } \\
\text { para el periodo } 2004-2005 \text {. Sus hallazgos } \\
\text { muestran la no existencia de una relación } \\
\text { estadística entre el resultado social corpo- } \\
\text { rativo y el resultado financiero. }\end{array}$ & Indeterminada. \\
\hline Lopes de Oliveira y Moneva (2013) & $\begin{array}{l}\text { Caso de estudio de las empresas Petrobras } \\
\text { S.A. y Repsol S.A. en el periodo 2002- } \\
2007 \text {. Los hallazgos muestran que las } \\
\text { inversiones realizadas por las empresas en } \\
\text { programas sociales y culturales mejoraron } \\
\text { resultados corporativos en los ámbitos } \\
\text { económico y financiero. }\end{array}$ & Positiva. \\
\hline Miras, Carrasco y Escobar (2014) & $\begin{array}{l}\text { Metaanálisis de } 86 \text { estimaciones extraí- } \\
\text { dos de setenta artículos publicados en } \\
\text { el periodo 2000-2012. Los hallazgos } \\
\text { muestran que la política ambiental afecta } \\
\text { el rendimiento financiero. }\end{array}$ & Positiva \\
\hline $\begin{array}{l}\text { Valenzuela, Jara-Bertin y Villegas } \\
\text { (2015) }\end{array}$ & $\begin{array}{l}\text { Estudio para } 55 \text { empresas no financieras } \\
\text { en el periodo } 2007-2012 \text { que cotizan en la } \\
\text { bolsa de Santiago. Los hallazgos muestran } \\
\text { que variables sociales, éticas, medioam- } \\
\text { bientales y de empleados, influyen posi- } \\
\text { tivamente en el desempeño financiero. }\end{array}$ & Positiva. \\
\hline Mahoney y Roberts (2017) & $\begin{array}{l}\text { Investigación para trecientas empresas } \\
\text { canadienses de propiedad pública en } \\
\text { el periodo 1996-1999. Los hallazgos, } \\
\text { usando la metodología de panel de datos, } \\
\text { mostraron que no existe una relación entre } \\
\text { los desempeños social y financiero. }\end{array}$ & Indeterminada. \\
\hline
\end{tabular}

Fuente: elaboración propia. 
Tabla 2.

Dimensiones y principios del Pacto Global

\begin{tabular}{|l|l|}
\hline \multicolumn{1}{|c|}{ Dimensiones } & \multicolumn{1}{|c|}{ Principios } \\
\hline Derechos humanos & $\begin{array}{l}\text { 1. Las empresas apoyan y respetan la protección de los derechos } \\
\text { humanos fundamentales reconocidos universalmente dentro de } \\
\text { su ámbito de influencia. } \\
\text { 2. Las empresas se deben asegurar de no ser cómplices de la } \\
\text { vulneración de los derechos humanos. }\end{array}$ \\
\hline Relaciones laborales & $\begin{array}{l}\text { 3. Las empresas deben apoyar la libertad de asociación y el } \\
\text { reconocimiento efectivo del derecho a la negociación colectiva. } \\
\text { 4. Las empresas deben apoyar la eliminación de toda forma de } \\
\text { trabajo forzoso o realizado bajo coacción. } \\
\text { 5. Las empresas deben apoyar la abolición efectiva del trabajo } \\
\text { infantil. } \\
\text { 6. Las empresas deben apoyar la abolición de las prácticas de } \\
\text { discriminación en el empleo y ocupación. }\end{array}$ \\
\hline Medio ambiente & $\begin{array}{l}\text { 7. Las empresas deberán mantener un enfoque preventivo que } \\
\text { favorezca el medio ambiente. } \\
\text { 8. Las empresas deben fomentar las iniciativas que promuevan } \\
\text { una mayor responsabilidad ambiental. } \\
\text { 9. Las empresas deben favorecer el desarrollo y la difusión de las } \\
\text { tecnologías respetuosas con el medio ambiente. }\end{array}$ \\
\hline Lucha contra la corrupción & $\begin{array}{l}\text { 10. Las empresas deben trabajar contra la corrupción en todas sus } \\
\text { formas, incluidas la extorsión y el soborno. }\end{array}$ \\
\hline
\end{tabular}

Fuente: Pacto Global Colombia (2017).

implementación y otra de comunicación (informes o reportes de RS).

\section{MATERIALES Y MÉTODOS}

Para calcular la relación entre el desempeño financiero y la pertenencia al PG, se usó como variable dependiente la rentabilidad de los activos y como variables independientes el PG, el tamaño de las empresas, la deuda de estas y el sector económico al que pertenecen.
Los datos se obtuvieron para las mil empresas más grandes de Colombia que reportaron información de sus balances y estados financieros a la Superintendencia de Sociedades en el 2017. La tabla 3 describe las variables con las que se trabajó.

Siguiendo la propuesta de Ortas, Álvarez y Garayar (2015), el modelo que se estimó por el método de Mínimos Cuadrados Ordinarios (MCO) fue de la forma:

$$
R O A=\beta_{0}+\beta_{1} P G+\beta_{2} \text { Tamaño }+\beta_{3} \text { Deuda }+\sum_{i=2}^{7} \beta_{4} \text { Sector }+\varepsilon_{i}
$$

Sin embargo, este modelo no arrojó estimadores MELI (Mejor Estimador Lineal Insesgado). Para corregir esta anomalía se volvió a hacer la estimación, pero usando el método de Mínimos Cuadrados Generalizados Factibles (MCGF). Por eso se estimó la ecuación (2), considerando los residuales al cuadrado de la ecuación (1). 
Tabla 3.

Variables del modelo

\begin{tabular}{|c|c|c|c|}
\hline $\begin{array}{l}\text { Tipo de } \\
\text { variable }\end{array}$ & Variable $^{\mathrm{a} /}$ & Descripción & $\begin{array}{c}\text { Variable usada } \\
\text { con anterioridad } \\
\text { por }\end{array}$ \\
\hline Dependiente & $\begin{array}{l}\text { Rentabilidad de los activos } \\
\text { (ROA) }\end{array}$ & $\begin{array}{l}\text { Cociente entre la utilidad anual } \\
\text { neta y los activos totales. } \\
\text { Media }=0,014 \text {. } \\
\text { Desv. estándar }=0,439\end{array}$ & $\begin{array}{l}\text { Makni, Fran- } \\
\text { coeur, y Bella- } \\
\text { vance (2009). }\end{array}$ \\
\hline \multirow[t]{4}{*}{ Independientes } & $\begin{array}{l}\text { Pertenencia al Pacto Global } \\
(\mathrm{PG})\end{array}$ & $\begin{array}{l}\text { Variables dummy: } \\
1=\text { Empresas adheridas al PG. } \\
0=\text { Empresas no adheridas al PG. } \\
\text { Media }=0,102 . \\
\text { Desv. estándar }=0,302 .\end{array}$ & n. a. \\
\hline & $\begin{array}{l}\text { Tamaño de la empresa } \\
\text { (variable de control) }\end{array}$ & $\begin{array}{l}\text { Variable proxy. } \\
\text { Se calculó sacando el logaritmo } \\
\text { natural a los activos totales. } \\
\text { Media }=19,39 \\
\text { Desv. estándar }=1,296 \\
\end{array}$ & $\begin{array}{l}\text { Valenzuela, Jara- } \\
\text { Bertin y Villegas } \\
(2015) \text {. }\end{array}$ \\
\hline & $\begin{array}{l}\text { Deuda de la empresa } \\
\text { (variable de control) }\end{array}$ & $\begin{array}{l}\text { Cociente entre pasivos y activos } \\
\text { totales. } \\
\text { Media }=0,623 \\
\text { Desv. estándar }=0,493\end{array}$ & $\begin{array}{l}\text { Valenzuela, Jara- } \\
\text { Bertin y Villegas } \\
(2015) \text {. }\end{array}$ \\
\hline & $\begin{array}{l}\text { Sector económico } \\
\text { (variable de control) }\end{array}$ & $\begin{array}{l}\text { Variable categórica. } \\
\text { Seis sectores: } \\
\text { 2= Agropecuario. } \\
3 \text { = Comercio. } \\
4 \text { = Construcción. } \\
\text { 5 = Manufactura. } \\
\text { 6= Minero. } \\
7 \text { = Servicios. }\end{array}$ & n.a. \\
\hline
\end{tabular}

a/ Utilidad anual neta, activos y pasivos totales medidos en pesos colombianos (COP).

Fuente: elaboración propia.

$u^{2}=\beta_{0}+\beta_{1} P G+\beta_{2}$ Tamaño $+\beta_{3}$ Deuda $+\sum_{i=2}^{7} \beta_{4}$ Sector $+v$

Una vez efectuado este procedimiento, se estimaron los valores esperados $\widehat{h}_{l}$, de los cuales se halló la raíz cuadrada para que dividieran cada valor de la matriz de varianzas y covarianzas, con lo cual se transformó el modelo en uno con errores homocedásticos.

\section{RESULTADOS Y DISCUSIÓN}

Los resultados usando los dos métodos descritos en la sección anterior, se presentan en la tabla 4. 
Tabla 4.

Resultados de las regresiones por los métodos de MCO y MCGF ${ }^{a /}$

\begin{tabular}{|l|c|c|}
\hline \multirow{2}{*}{ Constante } & \multicolumn{2}{|c|}{ ROA } \\
\cline { 2 - 3 } & MCO & MCGF \\
\hline \multirow{2}{*}{ PG } & $1,225^{* *}$ & $0,435^{* *}$ \\
& $(0,106)$ & $(0,006)$ \\
\hline Tamaño & 0,029 & $0,045^{* *}$ \\
& $(0,021)$ & $(0,001)$ \\
\hline Deuda & $-0,038^{* *}$ & $-0,012 * *$ \\
& $(0,005)$ & $(0,0003)$ \\
\hline \multirow{2}{*}{ Sector } & $-0,824 * *$ & $-0,309 * *$ \\
& $(0,012)$ & $(0,002)$ \\
\hline
\end{tabular}

Sector económico

\begin{tabular}{|l|c|c|}
\hline Comercio & $\begin{array}{c}0,077 * \\
(0,034)\end{array}$ & $\begin{array}{c}0,038^{* *} \\
(0,001)\end{array}$ \\
\hline Construcción & $\begin{array}{c}0,057 \\
(0,04)\end{array}$ & $\begin{array}{c}0,045^{* *} \\
(0,001)\end{array}$ \\
\hline Manufactura & $\begin{array}{c}-0,004 \\
(0,034)\end{array}$ & $\begin{array}{c}-0,017 * * \\
(0,001)\end{array}$ \\
\hline Minero & $\begin{array}{c}0,004 \\
(0,039)\end{array}$ & $\begin{array}{c}0,015^{* *} \\
(0,002)\end{array}$ \\
\hline Servicios & $\begin{array}{c}0,048 \\
(0,034)\end{array}$ & $\begin{array}{c}0,017 * * \\
(0,001)\end{array}$ \\
\hline Prueba F & $562,58 * *$ & \\
\hline Prueba Chi2 & & $38783,59 * *$ \\
\hline R2 & 0,8195 & \\
\hline N & 1000 & 708 \\
\hline
\end{tabular}

a/ *Significancia al $5 \%$; **significancia al $99 \%$; valores de los errores estándar entre paréntesis. Fuente: elaboración propia.

La regresión por el método de $\mathrm{MCO}$ mostró que el coeficiente de la ROA es positivo, pero no significativo estadísticamente. Resultado poco confiable, si consideramos que los estimadores presentaron problemas de heterocedasticidad, como lo muestran las pruebas de White y Breusch-Pagan/Cook-Weisberg (Wooldridge, 2006) que se encuentran en el anexo. En cambio, el método de MCGF arrojó un coeficiente significativo y positivo para la variable $\mathrm{PG}$, de donde se interpreta que el ROA mejora en 4,5 $\%$ para las empresas adheridas en comparación a las que no adhirieron.

Asimismo, las estimaciones por los métodos de MCO y MCGF muestran que las variables (de control) tamaño y deuda de la empresa afectan negativamente al roa.

En cuanto a los sectores económicos (variable de control), si una empresa hace parte del sector comercio, esta incrementa su ROA según ambos métodos de estimación. Para el resto de sectores, la lectura de los coeficientes es ambigua, porque según la estimación por MCGF, pertenecer a los sectores de construcción, minero y servicios incrementa el ROA, mientras que pertenecer al sector manufacturero lo reduce (coeficientes significativos al $99 \%$ ). En contraste, por MCO los coeficientes no son significativos a pesar de tener los mismos signos que los obtenidos por MCGF.

\section{CONCLUSIONES}

Existen varios estudios que han demostrado una relación positiva entre el desempeño financiero y la RS, otros como los de Makni, Francoeur y Bellavance (2009) y Mahoney y Roberts (2017) demuestran que la relación es indeterminada. Esto último coincide con los hallazgos aquí realizados, si se tiene en cuenta que los métodos de estimación del modelo no son concluyentes, dado que el cálculo usando MCO no muestra una relación que sea estadística significativa, mientras la estimación por MCGF sí lo hace. Esto deja en duda el vínculo 
entre las variables de las empresas colombianas que reportaron a la Superintendencia de Sociedades en el 2017.

Variables como la cultura podrían incorporarse a futuros estudios, como lo realizaron Miras, Carrasco y Escobar (2014) y Walman et al. (2006). Esto para el diseño de programas de RS según el contexto cultural de los países (comunidades, empresarios), con el fin de facilitar el cumplimiento de los Objetivos de Desarrollo Sostenible y por consiguiente de los principios del PG.

Estudios realizados en Colombia sobre la temática de este documento no se evidenciaron en la revisión efectuada, son investigadores para otros países los que han trabajado el tema desde perspectivas muy diversas. Así pues, se podría ahondar en esta línea de trabajo investigativo, haciendo uso de paneles de datos para determinar con más claridad si las compañías colombianas adheridas al PG cambian su desempeño financiero o no al hacer RS. Se podrían también cotejar otros indicadores financieros diferentes al ROA, como por ejemplo el ROE.

\section{REFERENCIAS BIBLIOGRÁFICAS}

Bour, E. (2012). Responsabilidad social de la empresa responsabilidad análisis del concepto. Estudios Económicos, 29(59), 1-30.

Coase, R. (1994). La empresa, el mercado y la ley. Madrid: Alianza Económica.

Comisión Europea (2001). Libro verde: fomentar un marco europeo para la responsabilidad social de las empresas. Informes, Comisión Europea, Empresa, Políticas Públicas,
RSC, Unión Europea. Recuperado de https://observatoriorsc.org/ libro-verde-fomentar-un-marcoeuropeo-para-la-responsabilidadsocial-de-las-empresas/

Demsetz, H. (1997). La economía de la empresa. Madrid: Alianza.

Duque, Y., Cardona, M. y Rendón, J. (2014). Responsabilidad social empresarial: teorías, índices, estándares y certificaciones. Cuadernos de Administración, 29(50), 196-206. doi:10.25100/cdea.v29i50.55.

Drucker, P. (1984). The new meaning of corporate social responsibility. $\mathrm{Ca}$ lifornia Management Review, 26(2), 53-63. doi: 10.2307/41165930.

Friedman, M. (1970). The social responsibility of business is to increase its profits. NY Times. Recuperado de http://www.colorado.edu/studentgroups/libertarians/issues/friedmansoc-resp-business.html

Liya, Z. y Shaohong, Y. (2013). The effect of corporate social responsibility on employees. 6th International Conference on Information Management, Innovation Management and Industrial Engineering, 268-271.

Lopes de Oliveira, M. y Moneva, J. (2013). El desempeño económico financiero y responsabilidad social corporativa Petrobras versus Repsol. Contaduría y Administración, 58(1), 131-167. doi: 10.1016/S01861042(13)71201-4.

Mahoney, L. y Roberts, R. (2007). Corporate social performance, financial performance and institutional ownership in Canadian firms. Accounting Forum, 31(3), 233-253. doi: 10.1016/j.accfor.2007.05.001. 
Makni, R. Francoeur, C. y Bellavance, F. (2009). Causality between corporate social performance and financial performance: evidence from canadian firms. Journal of Business Ethics, (89), 402-422. doi: 10.1007/ s10551-008-0007-7

Martínez, J., Prado, J. y Fernández, J. M. (2013). Responsabilidad social corporativa vs. Responsabilidad contable. Revista de Contabilidad, 16(1), 32-45. doi: 10.1016/S11384891(13)70004-9

Miras, M., Carrasco, A. y Escobar, B. (2014). Responsabilidad social corporativa y rendimiento financiero: un meta-análisis. Spanish Journal of Finance and Accounting, 43(2), 193-215.

Nieto, M. y Fernández, R. (2004). Responsabilidad social corporativa : la última innovación en management. Universia Business Review, 1, 2839. doi: 10.3145/epi.2011.may.15

Olcese, A., Rodríguez, M. y Alfaro, J. (2008). Manual de la empresa responsable y sostenible. Madrid: McGraw-Hill.

Ortas, E., Álvarez, I. y Garayar, A. (2015). The environmental, social, governance, and financial performance effects on companies that adopt the United Nations Global Compact. Sustainability, 7(2), 1932-1956.

Pacto Global Colombia (2017). Pacto global Colombia. Recuperado de http:// www.pactoglobal-colombia.org/

Preston, L. y O'Bannon, D. (1997). The corporate social-financial performance relationship: a typology and analysis. Business and Society, 36(5), 419-29. doi: 10.1177/000765039703600406

Valenzuela, L., Jara-Bertin, M. y Villegas, F. (2015). Prácticas de responsabilidad social, reputación corporativa y desempeño financiero. Revista de Administración de Empresas, 329-344.

Walman, D., et al. (2006). Cultural and leadership predictors of corporate social responsibility values of top management: a GLOBE study of 15 countries. Journal of International Business Studies, 37, 823-837. doi: 10.1057/palgrave.jibs. 8400230

Wooldridge, J. (2006). Introducción a la econometría. Madrid: Thompson.

Yepes, G., Peña, W. y Sánchez, L. (2007). Responsabilidad Social Empresarial. Bogotá: Universidad Externado de Colombia. 


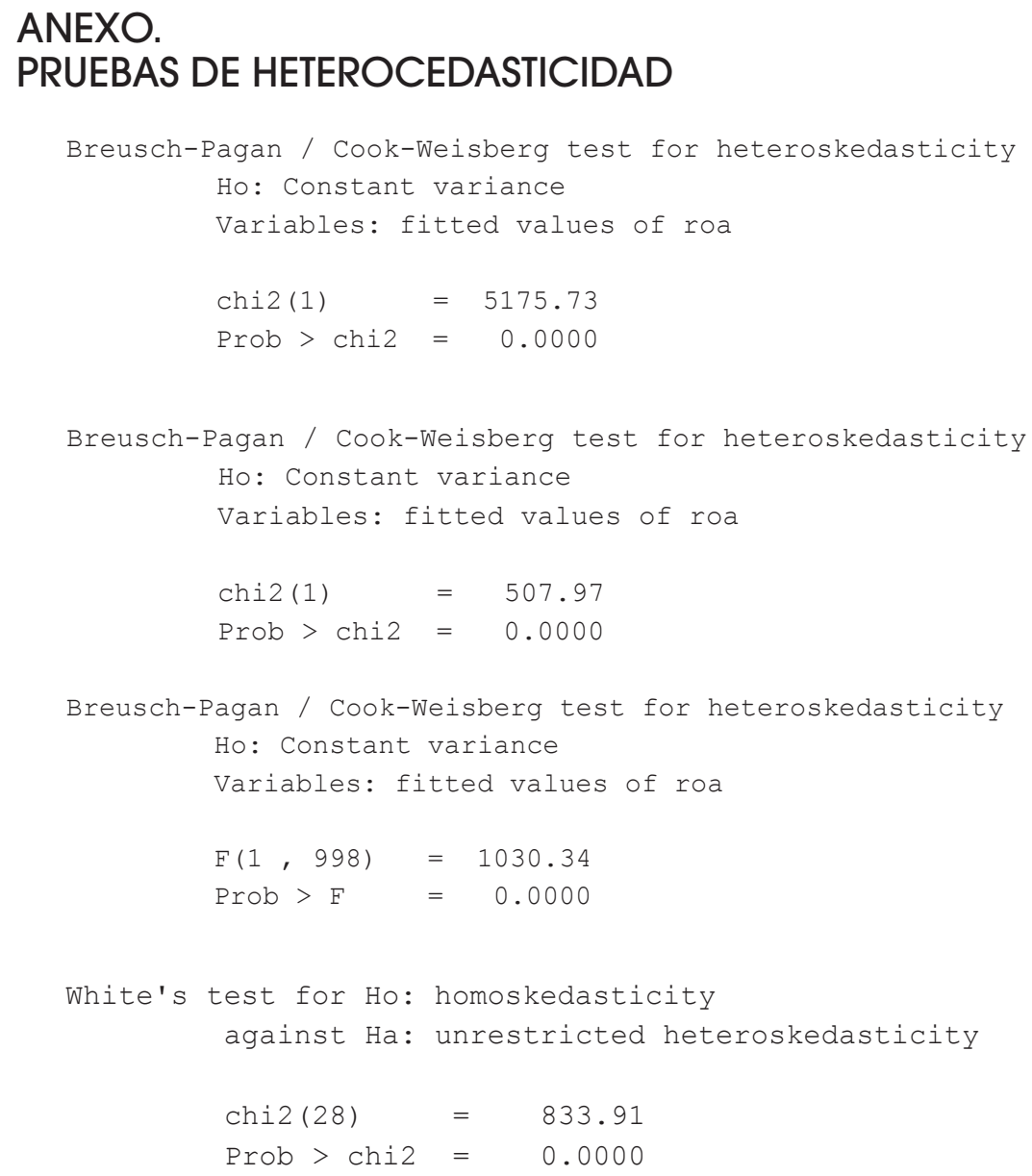

Fuente: elaboración propia 